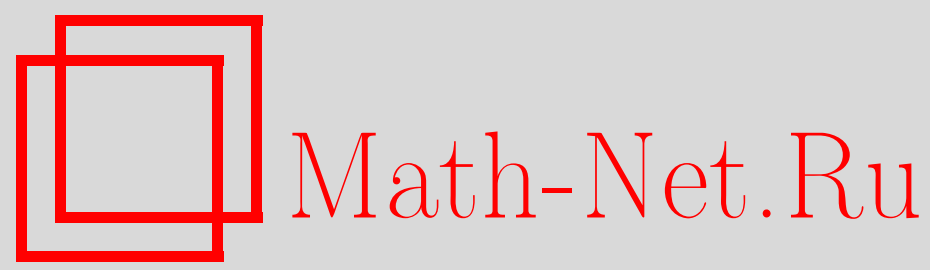

Н. Я. Медведев, Вложения в решеточно доупорядочиваемые группы, Матем. заметки, 2001, том 69, выпуск 5, 708712

DOI: https://doi.org/10.4213/mzm534

Использование Общероссийского математического портала Math-Net.Ru подразумевает, что вы прочитали и согласны с пользовательским соглашением http://www. mathnet.ru/rus/agreement

Параметры загрузки:

IP : 107.22 .136 .117

26 апреля 2023 г., 10:44:12

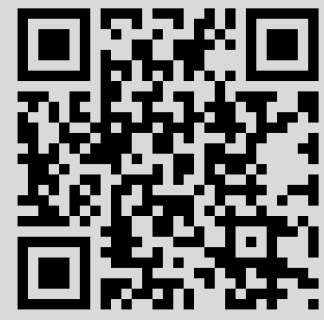




\title{
ВЛОЖЕНИЯ В РЕШЕТОЧНО ДОУПОРЯДОЧИВАЕМЫЕ ГРУППЫ
}

\author{
Н. Я. Медведев
}

\begin{abstract}
В работе получены следующие результаты: показано существование простых делимых решеточно доупорядочиваемых групп (теорема 2.1); доказано, что любая счетная решеточно упорядочиваемая или правоупорядочиваемая группа изоморфно вложимав простую делимую решеточно доупорядочиваемую группу (теорема 2.2, следствие 2.3 ).

Библиографоия: 10 названий.
\end{abstract}

Группа $G$ называется решеточно линейно упорядочиваемой (правоупорядочиваемой), если на ней можно определить решеточный (линейньй) порядок, устойчивьй относительно умножения справа и слева (умножения справа) на элементы группы $G$. Группа $G$ назьвается решеточно доупорядочиваемой, если любой ее частичньй порядок может быть продолжен до решеточного порядка. Группа $G$ называется делимой, если в ней разрешимы уравнения $x^{n}=g(n-$ натуральное число) для любого элемента $g \in G$. В работе получены следующие результаты: 1$)$ показано существование простых делимых решеточно доупорядочиваемых групп (теорема 2.1), 2) доказано, что любая счетная решеточно упорядочиваемая или правоупорядочиваемая группа изоморфно вложима в простую делимую решеточно доупорядочиваемую группу (теорема 2.2 , следствие 2.3).

1. Определения и вспомогательные результаты. Группа $G$ назьвается частично упорядоченной, если на ней определено отношение частичного порядка $\leqslant$, устойчивое относительно умножения слева и справа на элементы групшы $G$, т.е. для любых $x, y, z \in G$ если $x \leqslant y$, то $z x \leqslant z y$ и $x z \leqslant y z$. Хорошо известно, что любой частичный порядок групшы $G$ единственным образом определяется полугруппой положительных элементов и что любая чистая инвариантная полугруппа $P$ группы $G$ определяет единственньм образом частичньй порядок на групе $G$, при котором $P$ является полугруппой положительных элементов $[1$, с. 16$]$. Поэтому в дальнейшем мы определяем частичные порядки на группе либо задавая отношение $\leqslant$, либо определяя чистую инвариантную полугруппу. Все необходимые факты по упорядоченньм группам можно найти в [1]-[3], по теории групп - в [4]. Всюду в работе $\mathbb{R}$ обозначает естественно линейно упорядоченное множество действительных чисел, $\mathbb{N}, \mathbb{Z}$ - множества натуральных и целых чисел соответственно. Символ $S_{G}(M)$ обозначает инвариантную полугрупшу группы $G$, порожденную подмножеством $M$.

Работа выполнена при финансовой поддержке Российского фонда фундаментальных исследований, грант № 99-01-00156, и грантового центра НГУ Минобразования РФ, грант № 1. 
Пусть $A(\mathbb{R})$ - группа всех порядковых автоморфизмов линейно упорядоченного множества действительных чисел $\mathbb{R}$. Для любого $g \in A(\mathbb{R})$ положим $\operatorname{supp}(g)=\{x \in \mathbb{R} \mid$ $(x) g \neq x\}$. Рассмотрим в $A(\mathbb{R})$ нормальную подгруппу $L(\mathbb{R})$, состоящую из всех порядковых автоморфизмов $g(g \in A(\mathbb{R}))$, носитель $\operatorname{supp}(g)$ которых ограничен сверху. Свойства группы $A(\mathbb{R})$, ее подгрупп и, в частности, фактор-группа $A(\mathbb{R}) / L(\mathbb{R})$ рассматривались в работах [5]-[9]. В работах [5], [6] доказано, что группа $A(\mathbb{R}) / L(\mathbb{R})$ является делимой простой группой. В [8] получено полное описание всех максимальных и всех минимальных нетривиальных частичных порядков группы $A(\mathbb{R})$ и, в частности, доказано, что группа $A(\mathbb{R})$ имеет в точности два (взаимно обратных) различных решеточных порядка.

Напомним, что опорным интерваломо $(f, a)$ элемента $f \in A(\mathbb{R})$, содержащим $a \in \mathbb{R}$, назьвается множество тех $x \in \mathbb{R}$, для которых найдутся целые числа $m, n \in \mathbb{N}$ такие, что $(a) f^{n}<x<(a) f^{m}$. Опорньй интервал $o(f, a)$ состоит из одной точки $a$ лишш в том случае, если $(a) f=a$. В противном случае $f$ действует на $o(f, a)$ без неподвижных точек, т.е. если $(a) f>a$, то вьполнено $(x) f>x$ для любого $x \in o(f, a)(f$ действует на $o(f, a)$ положительно) или $(x) f<x$ для любого $x \in o(f, a)$ ( $f$ действует на $o(f, a)$ отрицательно).

ЛЕмма 1.1 (Холланд [5]). Элементы $f$ и $g$ группы $A(\mathbb{R})$ сопряжены в $A(\mathbb{R})$ тог-

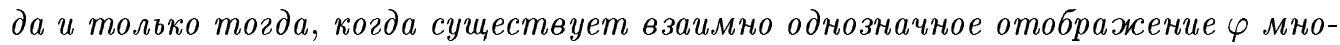
жества опорных интервалов $g$ на мнохество опорных интервалов $f$ такое, что если $о(g, a)<o(f, a)$, то $(o(g, a)) \varphi<(o(f, a)) \varphi$, и если $g$ действует на о $(g, a)$ полохительно (отрицательно или тождественно), то $f$ действует на $(o(f, a)) \varphi$ также положительно (отрицательно или тождественно).

Отметим также, что группа $A(\mathbb{R})$ является решеточно упорядоченной [2, с. 22] относительно порядка $P=\{f \in A(\mathbb{R}) \mid(x) f \geqslant x \forall x \in \mathbb{R}\}$ и подгруппа $L(\mathbb{R})$ является идеалом $[2$, с. 83$]$ в $l$-группе $A(\mathbb{R})$.

Хорошо известно, что множество всех частичных порядков в любой группе замкнуто относительно произвольных пересечений и, следовательно, образует полную нижнюю полурешетку относительно пересечения. Из леммы Цорна следует, что любой частичный порядок содержится в некотором максимальном частичном порядке. Те же самые утверждения выполняются и для изолированных частичных порядков группы. Тривиальньй частичньй порядок $P=\{e\}$ является наименьшим частичным порядком в любой группе. В общем случае минимальных нетривиальных частичных порядков может и не быть, как, например, в случае аддитивной группы целых чисел, но если они есть, то мощность множества всех минимальных нетривиальных частичных порядков либо четное натуральное число, либо бесконечна.

2. Основные результаты. Выберем действительное число $b$ такое, что $-\infty<b<$ $+\infty$. Положим $(x) q$ равной произвольной функции из грушы $A(\mathbb{R})$, удовлетворяющей следующим условиям:

1) $\operatorname{supp}(q)=(b,+\infty)$,

2) $(x) q>x$ для любого $x \in(b,+\infty)$.

Положим $Q=S_{A(\mathbb{R}) / L(\mathbb{R})}(q L(\mathbb{R}), e L(\mathbb{R}))$. Несложная проверка показьвает, что $Q$ является частичным порядком в группе $A(\mathbb{R}) / L(\mathbb{R})$.

Лемма 2.1. Частичный порядок $Q$ әруппы $A(\mathbb{R}) / L(\mathbb{R})$ является минимальным нетривиальным частичным порядком в группе $A(\mathbb{R}) / L(\mathbb{R})$. 
ДоКАЗАТЕЛЬСТВО. Пусть $f L(\mathbb{R})$ - произвольный элемент полугруппы $Q$. Тогда $f L(\mathbb{R})=q^{g_{1}} q^{g_{2}} \cdots q^{g_{n}} L(\mathbb{R})$ для некоторых $g_{1}, g_{2}, \ldots, g_{n} \in A(\mathbb{R})$, где $n \in \mathbb{N}$. Очевидно, что носитель $\operatorname{supp}\left(q^{g_{i}}\right)$ любого элемента $q^{g_{i}}(i=1, \ldots, n)$ состоит из одного интервала $\left(b_{i},+\infty\right)$. Но тогда очевидно, что носитель $q^{g_{1}} q^{g_{2}} \cdots q^{g_{n}}$ совпадает с некоторьм интервалом $\left(b_{i(0)},+\infty\right)$ для некоторого $1 \leqslant i(0) \leqslant n$ и $q^{g_{1}} q^{g_{2}} \cdots q^{g_{n}}$ действует положительно на опорном интервале $\left(b_{i}(0),+\infty\right)$. По лемме 1.1 элемент $q^{g_{1}} q^{g_{2}} \cdots q^{g_{n}}$ сопряжен с элементом $q$. Поэтому инвариантная полугруппа $S_{A(\mathbb{R}) / L(\mathbb{R})}(f L(\mathbb{R}), e L(\mathbb{R}))$ содержит полугруппу $Q$, что и доказьвает минимальность порядка $Q$.

ЛЕмма 2.2. Любой нетривиальный частичный порядок группы $A(\mathbb{R}) / L(\mathbb{R})$ содержит либо частичный порядок $Q$, либо частичный порядок $Q^{-1}$.

ДокАЗАТЕЛЬСТво. Пусть $X$-произвольньй частичный порядок групшы $A(\mathbb{R}) / L(\mathbb{R})$. Тогда $X \supseteq S_{A(\mathbb{R}) / L(\mathbb{R})}(f L(\mathbb{R}), e L(\mathbb{R}))$ для некоторого $f \in A(\mathbb{R}) \backslash L(\mathbb{R})$. Без ограничения общности можно считать, что носитель $\operatorname{supp}(f)$ ограничен снизу и содержит первьй опорньй интервал. Предположим также для определенности, что $f$ действует положительно на первом опорном интервале. $\operatorname{Ecли~} \operatorname{supp}(f)$ содержит опорньй интервал вида $(b,+\infty)$, то, очевидно, $X \subseteq Q$ или $Q^{-1}$. Если $\operatorname{supp}(f)$ не содержит опорного интервала вида $(b,+\infty)$, то $\operatorname{supp}(f)$ содержит строго возрастающую последовательность опорных интервалов $\left(a_{n}, b_{n}\right) \quad(n \in \mathbb{N})$ такую, что $\lim _{n \in \mathbb{N}} a_{n}=\lim _{n \in \mathbb{N}} b_{n}=+\infty$ и $f$ действует одновременно на всех опорных интервалах $\left(a_{n}, b_{n}\right) \quad(n \in \mathbb{N})$ положительно или отрицательно. Предположим, что $f$ действует положительно. Повторяя рассуждения, проведенные в работе [8] при доказательстве предложения 13, получаем, что инвариантная полугруппа $S_{A(\mathbb{R})}(f, e)$ содержит элемент $q$.

Пусть теперь $f$ действует одновременно на всех опорных интервалах $\left(a_{n}, b_{n}\right)(n \in \mathbb{N})$ отрицательно. Тогда рассуждения, проведенные в работе [8] при доказательстве предложения 13, показьвают, что инвариантная полугрупша $S_{A(\mathbb{R})}(f, e)$ содержит элемент $q_{2}$ такой, что:

1) $\operatorname{supp}\left(q_{2}\right)=(\alpha, \beta) \cup(\gamma,+\infty)$,

$2)$ на интервале $(\alpha, \beta)$ элемент $q_{2}$ действует положительно,

$3)$ на интервале $(\gamma,+\infty)$ элемент $q_{2}$ действует отрицательно.

В этом случае ясно, что $X \supseteq S_{A(\mathbb{R}) / L(\mathbb{R})}(f L(\mathbb{R}), e L(\mathbb{R}))$ содержит элемент $q^{-1}$ и, следовательно, частичньй порядок $Q^{-1}$. Оставшиеся случаи рассматриваются аналогично.

СлЕДСТВИЕ 2.1. Любой минимальный частичный порядок группы $A(\mathbb{R}) / L(\mathbb{R})$ совпадает либо с порядком $Q$, либо с порядком $Q^{-1}$.

Лемма 2.3. Пусть $M_{1}$ и $M_{2}$-максимальные частичные порядки группь $A(\mathbb{R}) /$ $L(\mathbb{R})$ и порядки $M_{1}$ и $M_{2}$ содерәст минимальный порядок $Q\left(Q^{-1}\right)$. Тогда $M_{1}=M_{2}$.

ДоКАЗАТЕЛЬСТво. Предположим, что порядки $M_{1}$ и $M_{2}$ содержат минимальный порядок $Q$. Пусть $g L(\mathbb{R}) \in M_{1} \backslash M_{2}$. Тогда $S_{A(\mathbb{R}) / L(\mathbb{R})}\left(M_{2}, g L(\mathbb{R})\right)$ не является частичным порядком. Следовательно, существует $m L(\mathbb{R}) \in M_{2}$ такой, что $m L(\mathbb{R})=\left(g^{f_{1}}\right)^{-1} \times$ $\left(g^{f_{2}}\right)^{-1} \cdots\left(g^{f_{n}}\right)^{-1} L(\mathbb{R})$ для некоторых $f_{1}, \ldots, f_{n} \in A(\mathbb{R})$.

Тогда по лемме 2.2 для некоторого $\varepsilon= \pm 1$ выполняется

$$
Q^{\varepsilon} \subseteq S_{A(\mathbb{R}) / L(\mathbb{R})}(m L(\mathbb{R}), e L(\mathbb{R})) \subseteq S_{A(\mathbb{R}) / L(\mathbb{R})}\left(g^{-1} L(\mathbb{R}), e L(\mathbb{R})\right) \subseteq M_{2} .
$$

Отсюда непосредственно следует, что $Q^{\varepsilon} \subseteq M_{1}^{-1}, M_{2}$, а это невозможно по нашему предположению. 
СлЕДСТВИЕ 2.2. Группа $A(\mathbb{R}) / L(\mathbb{R})$ имеет не более двух максимальных частичных порядков.

TEOPEMA 2.1.

1) Простая группа $A(\mathbb{R}) / L(\mathbb{R})$ имеет в точности два максимальных частичных порядка.

2) Оба эти частичных порядка являются решеточными.

3) Любой частичный порядок группы $A(\mathbb{R}) / L(\mathbb{R})$ продолжсается до решеточного порядка.

ДокАЗАтЕльСтво. Рассмотрим в группе $A(\mathbb{R}) / L(\mathbb{R})$ подмножество

$$
\begin{aligned}
R^{+}= & \{g L(\mathbb{R}) \mid g \in A(\mathbb{R}) \&(\exists \alpha \in \mathbb{R})(\alpha) g>\alpha \\
& \&(\forall \beta \in \mathbb{R}) \beta \geqslant \alpha \Longrightarrow(\beta) g \geqslant \beta\} .
\end{aligned}
$$

Непосредственная проверка показывает, что подмножество $R^{+}$является частичным порядком в групше $A(\mathbb{R}) / L(\mathbb{R})$. Заметим, что этот порядок максимален. Рассмотрим элемент $f \in A(\mathbb{R}) \backslash L(\mathbb{R})$. Если $f L(\mathbb{R}) \in\left(R^{+}\right)^{-1}$, то, очевидно, не существует частичного порядка, содержащего $R^{+} \cup\{f L(\mathbb{R})\}$. Поэтому считаем, что $f L(\mathbb{R}) \notin R^{+} \cup\left(R^{+}\right)^{-1}$. Но тогда элемент $f$ обладает следующими свойствами:

1) носитель $\operatorname{supp}(f)$ элемента $f$ содержит строго возрастающие последовательности опорных интервалов $\left(a_{n}, b_{n}\right)(n \in \mathbb{N})$ и $\left(c_{n}, d_{n}\right)(n \in \mathbb{N})$ такие, что

$$
\lim _{n \in \mathbb{N}} a_{n}=\lim _{n \in \mathbb{N}} b_{n}=\lim _{n \in \mathbb{N}} c_{n}=\lim _{n \in \mathbb{N}} d_{n}=+\infty
$$

2) на интервалах $\left(a_{n}, b_{n}\right)$ элемент $f$ действует положительно, а на интервалах $\left(c_{n}, d_{n}\right)$ элемент $f$ действует отрицательно.

Теперь рассуждения, аналогичные доказательству теоремы 17 из [8], показьвают, что произведение некоторых элементов, сопряженных с элементом $f L(\mathbb{R})$ в групше $A(\mathbb{R}) /$ $L(\mathbb{R})$, является неединичным элементом, лежашим в полугруппе $\left(R^{+}\right)^{-1}$. Поэтому и в этом случае не существует частичного порядка, содержащего $R^{+} \cup\{f L(\mathbb{R})\}$. Порядок $R^{+}$является решеточным, поскольку он является $\ell$-гомоморфньм образом естественно решеточно упорядоченной групшы $A(\mathbb{R})$ по идеалу $L(\mathbb{R})$. Оставшиеся утверждения очевидны.

Теорема 2.2. Любая счетная решеточно упорядочиваемая группа изоморфно вложима в простую делимую решеточно доупорядочиваемую группу.

ДокАЗАТЕЛЬСтво. Пусть $G$ - счетная решеточно упорядоченная группа. Тогда, как показано в книге [10, следствие $2 \mathrm{~L}$, с. 41$]$, любая счетная $l$-группа изоморфна $l$-подгруппе порядковых автоморфизмов $A(\mathbb{Q})$ линейно упорядоченного множества рациональных чисел $\mathbb{Q}$. Хорошо известно $[2$, с. 64$]$, что $A(\mathbb{Q})$ изоморфно вложима в $l$-группу $A(\mathbb{R})$. Поскольку линейно упорядоченное множество $\mathbb{R}$ порядково изоморфно открытому отрезку $(0,1)$, то можно считать, что $G$ является $l$-подгруппой $l$-группы всех порядковых автоморфизмов замкнутого интервала $[0,1]$, причем $(0) g=0$ и $(1) g=1$. Пусть $g$ - произвольньй элемент группы $G$. По элементу $g$ определим отображение $\bar{g}: \mathbb{R} \rightarrow \mathbb{R}$ линейно упорядоченного множества $\mathbb{R}$ на себя по правилу: $(x+k) \bar{g}=(x) g+k(x \in[0,1]$, $k \in \mathbb{Z})$. 
Несложная проверка показьвает, что $g \in A(\mathbb{R})$ и что $l$-подгруппа $\bar{G}$ решеточно упорядоченной группы $A(\mathbb{R})$, порожденная элементами $\{\bar{g} \mid g \in G\}, l$-изоморфна $l$-групе $G$. Поскольку $\bar{G} \cap L(\mathbb{R})=e$, то $l$-групша $\bar{G}$ и, следовательно, $l$-групша $G$ изоморфно вложима в фактор-группу $A(\mathbb{R}) / L(\mathbb{R})$. Теперь применение теоремы 2.1 завершает доказательство.

СЛЕДСТВИЕ 2.3. Любая счетная решеточно правоупорядочиваемая группа изоморфно вложсма в простую делимую решеточно доупорядочиваемую группу.

ДокАЗАТЕльство. Действительно, любая счетная правоупорядочиваемая группа изоморфно вложима в счетную решеточно упорядочиваемую группу [1, теорема 3.4.1]. Непосредственное применение теоремы 2.2 завершает доказательство.

\section{СПИСОК ЦИТИРОВАННОЙ ЛИТЕРАТУРЫ}

[1] Копытов В. М., Медведев Н. Я. Правоупорядоченные группы. Новосибирск: Научная книга (НИИ МИОО НГУ), 1996.

[2] Копытов В. М. Решеточно упорядоченные группы. М.: Наука, 1984.

[3] Kopytov V.M., Medvedev N. Ya. The Theory of Lattice-Ordered Groups. DordrechtBoston-New York: Kluwer Acad. Publ., 1994.

[4] Каргаполов М.И., Мерзляков Ю. И. Основы теории групп. М.: Наука, 1977.

[5] Holland C. W. The lattice-ordered group of automorphisms of an ordered set // Michigan Math. J. 1963. V. 10. P. 399-408.

[6] Lloyd J. T. Lattice-Ordered Groups and $o$-Permutation Groups. Ph. D. Thesis. New Orleans: Tulane University, 1964.

[7] Gurevich Y., Holland C. W. Recognizing the real line // Trans. Amer. Math. Soc. 1981. V. 265. P. 527-534.

[8] Holland C. W. Partial orders of the group of automorphisms of the real line // Contemp. Math. 1992. V. 137. P. 197-207.

[9] Giraudet M., Truss J. K. On distinguishing quotients of ordered permutation groups // Quart. J. Math. Oxford. 1994. V. 45. P. 181-209.

[10] Glass A. M. W. Ordered Permutation Groups. London Math. Soc. Lect. Notes. Ser. V. 55. Cambridge: Univ. Press, 1981. 